Chapter 4

\title{
Hybrid AHP and TOPSIS Methods Based \\ Cell Selection (HATCS) Scheme for Mobile WiMAX
}

\author{
Mohammed A. Ben-Mubarak, \\ Borhanuddin Mohd. Ali, Nor Kamariah Noordin, \\ Alyani Ismail and Chee Kyun Ng
}

Additional information is available at the end of the chapter

http://dx.doi.org/10.5772/55894

\section{Introduction}

In the past, the earlier cellular systems concentrated on voice calls as the main application that has to be considered to fulfil end-user requirements. However, nowadays with the variety of the user application and their requirements, the new 3G/4G systems have to consider many applications such as voice calls video streaming/conference, online gaming, peer-to-peer application and many other application and their differentrequirementsas shownin Table1 [1-2].

\begin{tabular}{|c|c|c|c|c|}
\hline & Application & Type & Bandwidth & Delay \\
\hline 1 & Multiplayer Interactive Gaming & Real-time & $50-85 \mathrm{kbps}$ & $<100 \mathrm{~ms}$ \\
\hline \multirow[t]{2}{*}{2} & VolP \& Video Conference & Real-time & 4-64 kbps (VolP) & $<150 \mathrm{~ms}$ \\
\hline & & & 32-384 kbps (Video call) & \\
\hline \multirow[t]{3}{*}{3} & Streaming Media & Real-time & 5-128 kbps (music) & $<300 \mathrm{~ms}$ \\
\hline & & & 20-348 kbps (video clip) & \\
\hline & & & "/>2 Mbps (movie streaming) & \\
\hline \multirow[t]{2}{*}{4} & Web Browsing \& Instant Messaging & Non & $<250$ kbps (instant messaging) & $\mathrm{N} / \mathrm{A}$ \\
\hline & & real-time & "/>500 kbps (email/web browsing) & \\
\hline 5 & Media Content Downloads & Non & "/>1 Mbps & N/A \\
\hline & & real-time & & \\
\hline
\end{tabular}

Table 1. WiMAX Application Types 
Although Mobile WiMAX promises to deliver the triple play services, handover mechanism still has some unsolved issues, which may affect the end user QoS requirements [3]. Cell selection is one of the main phases which may affect the user requirements after the handover process. After the Serving Base Station (SBS) advertisement message, MOB_NBR-ADV and the MS handover scanning, the MS will examine the collected PHY information on the neighbour BSs (nBSs) channel conditions to select the Target Base Station (TBS) for the coming handover [4]. The conventional cell selection scheme in Mobile WiMAX is based on signal quality, i.e. the nBS which has the best signal quality in terms of Received Signal Strength, will be considered as the TBS for the coming handover [5]. However, a single criterion like signal quality is not sufficient as a basis to choose the best BS for different end user application's requirements. As an illustration in Figure 1, suppose the MS is in an overlapping area of two or more BSs that have similar signal quality, there will be an ambiguity on which one will the MS choose for different user application requirements. Putting a cell selection criterion on signal quality entirely may make an MS choose a TBS with a good signal quality but one which may incur higher delay or smaller bandwidth, this may affect some real-time applications such as VoIP, video conferencing, streaming media, multiplayer interactive gaming.

Handover operation in Mobile WiMAX can be divided into two main phases; the pre-handover phase, and the actual handover phase. In the pre-handover phase the cell-reselection algorithm is one of the most important steps and this may affect the end-user QoS after the handover. On the other hand, the actual handover phase mainly focuses on handover execution and network re-entry [4]. Most of the published works are on the actual handover phase, about how to execute the scheme with a small number of messages [6-10], while there are only a few researches that focus on cell selection scheme.

The 802.16 standard [6] defines the receive signal strength indicator (RSSI) or carrier-tointerference plus noise ratio (CINR) as the handover trigger factor and cell selection scheme whereas WiMAX supports many multimedia data services therefore, it is not sufficient to let the signal strength be the only reference criteria.

In [11], the authors introduced an enhanced handover target cell selection algorithm for WiMAX network based on the effective capacity estimation and neighbour advertisement. The algorithm let each BS to estimate its idle capacity and broadcasts its effective idle capacity via the media access control (MAC) management message, MOB NBRADV, to help each MS to select the handover target cell. Therefore, the cell selection algorithm uses two criteria, idle capacity and signal strength as a weighted cost function to select the target cell. The result shows that this scheme enhances performance in terms of packet loss ratio and system throughput. Nevertheless, other criteria can be taken into account to provide better services for different user applications. In vertical handover schemes, i.e. handover between different network technologies, some researchers have introduced multi-criteria based network selection as described in [12-13].

Because of the increasing number of mobile stations (MS) and the support of high speed Internet and multimedia data services, the base station (BS) of WiMAX often works at a data rate close to maximum capacity. Therefore, it is not proper to let the signal strength to be the only reference criteria to choose the next cell. The MSs should decide intelligently to switch to 
another idle cell to get the best channel. In order to select a TBS that best meets the end-user application requirements this paper proposes a smart way for selecting the right TBS based on a set of multiple decision criteria. The method uses Analytic Hierarchy Process (AHP) method for criteria weighting and Technique for Order Preference by Similarity to Ideal Solution (TOPSIS) for the TBS ranking based on some criteria such as CINR, queue length, and bandwidth.

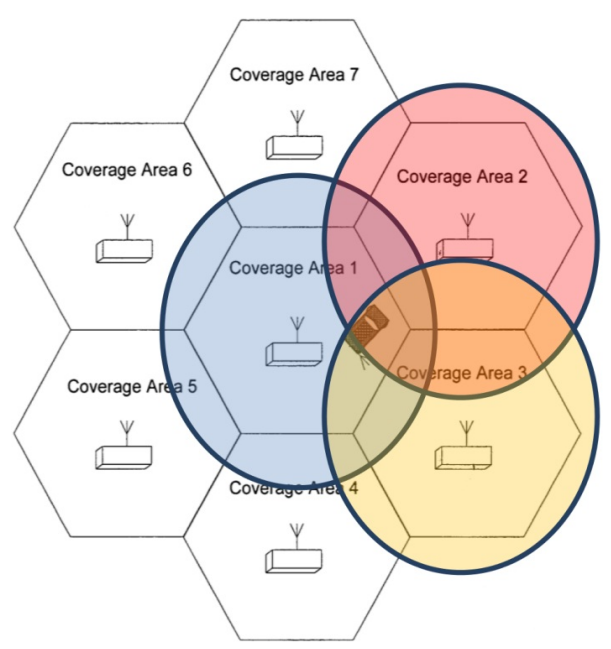

Figure 1. Cell selection

\section{Handover cell selection criteria}

The conventional scheme of cell selection in Mobile WiMAX is based on a single criterion which is signal quality. The nBS which has the best signal quality (ex. RSSI or CINR) will be considered as the TBS for the coming handover [5-6]. As highlighted earlier, this is inefficient because besides the signal quality each user's application has their own respective QoS requirements.

In this paper, two types of application are studied; VoIP as an example of real-time applications, and Media Content Downloads as an example of non real-time application [2]. As shown in Table 1, VoIP is a latency or delay sensitive application with low data rate demand. On the other hand, Media Content Download is a delay-tolerant application and generally demand high bandwidth. Thus, there are some other criteria that need to be considered when the cell selection decision is to be taken. In this paper, three criteria will be considered; they are CINR, $\mathrm{BW}$, and congestion delay.

Carrier-to-Interference-plus-Noise-Ratio (CINR): The signal quality is the main criteria for choosing the TBS, but this is not a sufficient criterion. 
Bandwidth (BW): This metric refers to the available bandwidth in WiMAX cell. It is simply the difference between the total capacities and the aggregated used BW in Kbps.

Congestion delay: This is the delay of packets due to queuing until they can be processed.

\section{Multi-criteria decision making methods}

The cell selection problem is about selecting one BS for handover among limited number of candidate BSs with respect to a set of different criteria. This is a typical Multiple Criteria Decision Making (MCDM) problem. In the study of decision making, terms such as MCDM is the problem of choosing an alternative solution from a set of alternatives, which are characterized in terms of their attributes [14]. The most popular classical MCDM methods are:

1. WSM (Weighted Sum Model): the overall score of a candidate BS is determined by the weighted sum of all the attribute values.

2. TOPSIS (Technique for Order Preference by Similarity to Ideal Solution): the chosen candidate $\mathrm{BS}$ is the one which is closest to ideal solution and the farthest from the worst case solution.

3. AHP (Analytic Hierarchy Process): decomposes the BS selection problem into several subproblems and assigns a weight value for each sub-problem.

In this paper, we use TOPSIS as alternative score ranking based cell selection scheme and compare it with WSM, while AHP will be used for weighting the attributes or the criteria based on the importance of each criterion for the end users application.

Cell selection could be considered as an MCDM problem. For instance, suppose a user is currently connected to BS A1 and has to make a decision among two alternative candidate BSs: A2 and A3. Handover criteria considered here are CINR, BW, and congestion delay, which are denoted as: $\mathrm{X} 1, \mathrm{X} 2$ and $\mathrm{X} 3$, respectively. The decision problem can be modelled in a decision matrix Dm as shown in (1), where the capabilities of each candidate are presented.

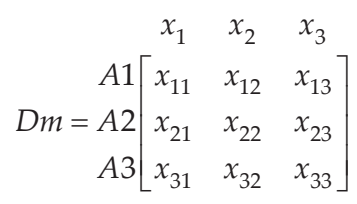

Where $x_{11}$ is the CINR, $x_{12}$ is the BW and $x_{13}$ is the congestion delay of BS A1. In similar way, $x_{21}, x_{22}$ and $x_{23}$ are the CINR, BW and congestion delay values of BS A2. In addition, $x_{31}, x_{32}$ and $x_{33}$ are the CINR, BW and congestion delay of BS A3.

Assume the user is using two types of applications; real-time application such as VoIP and non-real-time application such as Media Content Download. The traffic or application 
preference on handover criteria is modelled as weights assigned by the user on the criteria; for VoIP and Media Content Download which are shown in (2) and (3).

$$
\begin{gathered}
W r=\left[\begin{array}{lll}
w_{r 1} & w_{r 2} & w_{r 3}
\end{array}\right] \\
W n r=\left[\begin{array}{lll}
w_{n r 1} & w_{n r 2} & w_{n r 3}
\end{array}\right]
\end{gathered}
$$

The CINR and congestion delay are considered as important for voice application because as studied by [15-16], to get a minimum jitter and delay the CINR has to be good enough, while the bandwidth and CINR are considered important for the non real-time application such as Media Content Download.

\subsection{WSM (Weighted Sum Model)}

WSM is the most popular multi criteria decision making (MCDM) method. It is the simplest way of evaluating the number of alternatives $(m)$ in terms of a number of decision criteria $(n)$ [17]. The overall score of an alternative is calculated as the weighted sum of all the attribute values as shown in equation (4).

$$
A_{i}^{(W S M)}=\sum_{j}^{n} w_{j} x_{i j}, i=1,2,3, \ldots \ldots . . .
$$

Where $A i$ is the evaluated score of an alternative, $W j$ is the weight value for criteria $j, n$ number of criteria. Because the decision matrix value could be in different scales such as BW could be $10 \mathrm{Mbps}$ and the cell load could be $50 \%$ or 0.50 the decision matrix has to have a comparable scale (normalized) by using (5) for the benefit criteria (i.e. stronger CINR, larger BW) and (6) for cost criteria (i.e. more delay). In (5) and (6) $x_{i j}$ is the performance score of alternative $A_{i}$ with respect to criterion $x_{j}$ and $r_{i j}$ is the normalization value of $x_{i}$.

$$
\begin{aligned}
& r_{i j}=x_{i j} / x_{j} \text { MAX }, i=1, \ldots m, j=1, \ldots . n \\
& r_{i j}=x_{j M I N} / x_{i j}, i=1, \ldots m, j=1, \ldots . n
\end{aligned}
$$

\subsection{Technique for Order Preference by Similarity to Ideal Solution (TOPSIS)}

TOPSIS is one of MCDM methods based on the concept that the chosen alternative should have the shortest distance from the positive ideal solution (PIS) and the farthest from the negative ideal solution (NIS) for solving a multiple criteria decision making problem. Briefly, 
PIS is made up of all best values attainable criteria, whereas NIS is composed of all worst values incurred from criteria [14]. The calculation processes of this method are as follows. Normalize the decision matrix.

$$
r_{i j}=x_{i j} / \sqrt{\sum_{i=1}^{m} x_{i j}}, i=1, \ldots, m, j=1, \ldots n
$$

- Decision matrix is weighted using the weighting factor.

- Determine the ideal solutions $A^{+}$and the negative-ideal solutions $A^{-}$.

$$
\begin{aligned}
& A^{+}=\left(\max _{i} v_{i j} \mid j \in J\right),\left(\min _{i} v_{i j} \mid j \in J^{\prime}\right) \\
& A^{-}=\left(\min _{i} v_{i j} \mid j \in J\right),\left(\max _{i} v_{i j} \mid j \in J^{\prime}\right)
\end{aligned}
$$

Where $v_{i}$ is the weighted and normalized of the $x_{i j}$, while $J$ is associated with the benefit criteria and $J$ ' is associated with the cost criteria.

- Calculate the separation of each alternative from the ideal solution, and the negative ideal solution.

$$
\begin{aligned}
& S_{i+}=\sqrt{\sum_{j=1}^{m}\left(a_{i j}-a_{j+}\right)^{2}}, i=1, \ldots, n \\
& S_{i-}=\sqrt{\sum_{j=1}^{m}\left(a_{i j}-a_{j-}\right)^{2}}, i=1, \ldots, n
\end{aligned}
$$

- Relative closeness to the ideal solution is calculated.

$$
C_{i+}=S_{i-} /\left(S_{i-}+S_{i+}\right), i=1, \ldots, n
$$

\subsection{Analytic Hierarchy Process (AHP)}

The Analytic Hierarchy Process (AHP) is a structured technique for organizing and analyzing complex decision problems. It has a variety of applications used around the world in different fields of decision situations such as business, industry, healthcare, engineering and education.

It provides a comprehensive and rational framework for structuring a decision problem, for weighting the decision criteria, and for evaluating alternative solutions [14, 18]. In this paper, we use the AHP method for weighting the decision criteria. The calculation processes of this method are as follows and shown in Figure 2. 
The AHP method main steps are as follows:

1. Define the decision criteria and decompose the decision problem into different levels of the hierarchy.

2. Compare each factor to all the other factors within the same level through pairwise comparison matrix. The judgments in the AHP are made in pairs $a_{i j}$, relating the importance of criterion $i$ to that of criterion $j$. The criteria are compared pair-wise with respect to the goal. $A$ is a matrix indicating the importance of criterion $i$ relative to criterion $j$ as shown in Equation (12). Noticeably, the $a_{i j}=1$ when $i=j$, while $a_{j i}=1 / a_{i j}$, which reflects the reciprocal importance of criterion $j$ relative to criterion $i$.

$$
A=\left[\begin{array}{ccccc}
a_{11} & a_{12} & a_{13} & \ldots & a_{1 j} \\
a_{21} & a_{22} & a_{23} & \ldots & a_{2 j} \\
\cdot & & & & \\
a_{i 1} & a_{i 2} & a_{i 3} & \ldots & a_{i j}
\end{array}\right]
$$

1. After creating matrix $A$ of comparison, the next step is to determine the weights of the criteria, in which $w_{i}$ is the weight of objective $i$ in the weight vector $w=\left[w_{1}, w_{2}, \ldots, w_{n}\right]$ for $n$ criteria. To get this, the eigenvector is used.

The eigenvector can be calculated using the following steps:

1. Raise the $A$ matrix to powers square.

2. Sum each column of the squared matrix.

$$
a_{i}^{\prime}=\sum_{j=1}^{n} a_{i j}
$$

1. Then we divide each element of the matrix with the sum of its column, we have normalized relative weight. The sum of each column is 1 as shown in (14).

$$
a^{\prime \prime}=\frac{a_{i j}}{a_{i}^{\prime}}
$$

2. Check the Consistency Index (CI). The CI can be computed as the difference between the current and previous computed eigenvectors.

3. Previous steps are repeated until we get a very small value of CI.

4. Determine the weights of the criteria 


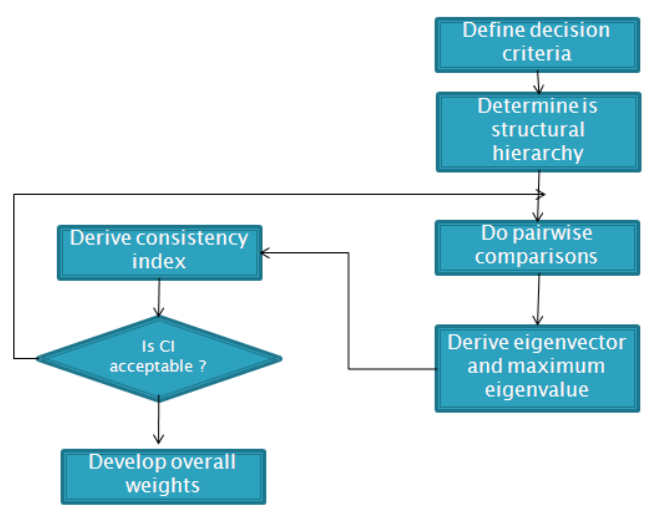

Figure 2. AHP calculation processes

\section{Hybrid AHP and TOPSIS based cell selection}

The conventional cell selection scheme in Mobile WiMAX is only based on the received signal strength. However, it is not sufficient to meet the different user application requirements that WiMAX promises to meet. The proposed scheme considers multiple criteria; CINR, BW and congestion delay for cell selection using some MCDM methods, AHP and TOPSIS. As shown in Figure 3, the proposed cell selection scheme can be divided into three main functions: "collecting info" which collects the decision criteria and network conditions, "criteria weighting" which processes criteria weighting using AHP methods based on application QoS requirements, and "alternatives ranking" which finalizes the process of cell selection using TOPSIS method.

\subsection{Collecting Information stage}

In this block the MS with BS assistance try to collect the information about some criteria that will help the cell selection decision. As highlighted earlier, this paper proposes three criteria decision, viz; CINR, BW and Congestion delay.

For the CINR criteria, the SBS regularly broadcast information about the nBSs through the neighbour advertisement messages (MOB_NBR-ADV). Additionally, the MS and the SBS can retrieve more information about the nBSs after the MS finished scanning, such as CINR and RSSI. The CINR is defined as the power ratio between a carrier and the interference and noise power [5].

$$
C I N R=10 \log _{10} \frac{C_{r}}{P_{I}+P_{N}}
$$




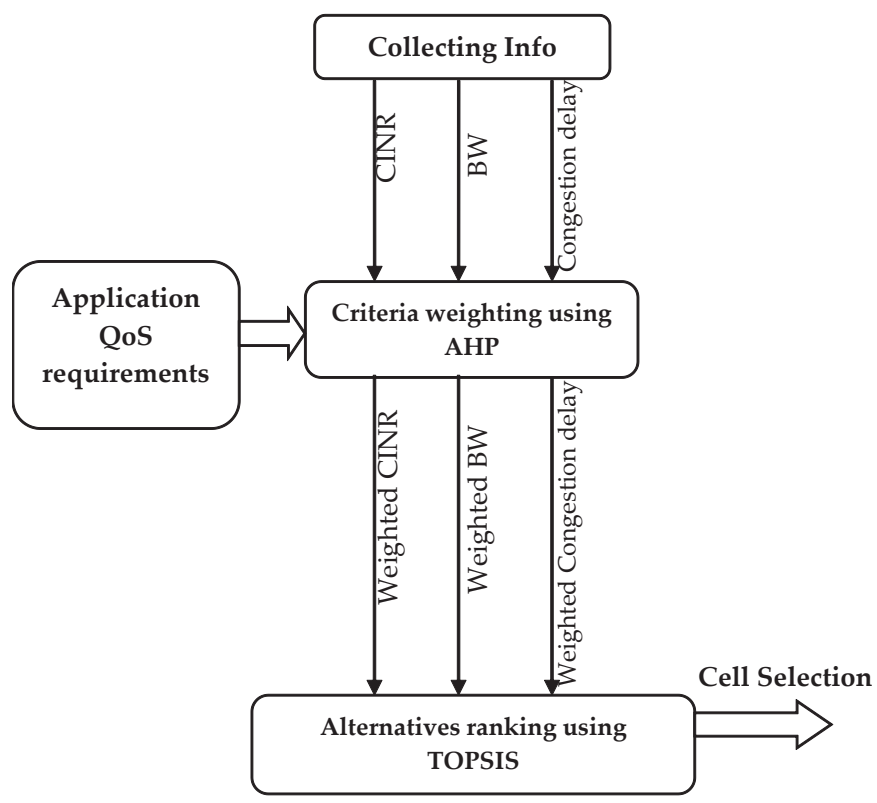

Figure 3. The proposed cell selection scheme

Where, $C_{r}$ is the received carrier power, $P_{I}$ is the interference power and $P_{N}$ is the noise power.

The available BW in WiMAX cell metric can be estimated based on [11]; after scanning process, the MS can exploit the information element (IE) in the DL-MAP/UL-MAP messages and aggregate the number of the allocated downlink and uplink physical slots (PSs). The available downlink BW can be estimated as follows;

$$
B W_{D L}(t)=\frac{1}{\tau} S_{D L-f r e e}(t-\tau, t) \quad C_{D L-s l o t}(t)
$$

Where $\tau$ is the period of time that we can get number of frame, $S_{D L}$-free is the number of unused PSs within $\tau$ while the $C_{D L-s l o t}$ is the number of bits that can be transmitted in one downlink PS.

Every nBS will inform the SBS its congestion delay through the backbone, and the SBS will send these values to the MS by the advertisement messages (MOB_NBR-ADV).

\subsection{Weighting criteria using AHP}

AHP is used to determine the weights for the three criteria -- CINR, BW and delay. It was reported that classical MADM methods cannot efficiently handle a decision problem with imprecise data that decision criteria could contain [14]. 
For that, the use of fuzzy logic can be used to deal with imprecise information and combine and evaluate multiple criteria simultaneously. Hence, fuzzy logic concept provides a robust mathematical framework in which handover decision can be formulated as a Fuzzy MADM.

The scale used is represented by the intensities between each other according to the fundamental scale. The fundamental scale is validated according to effectiveness and theoretical justifications according to [18]. The scale consists of nine levels. To make it even easier to judge, one can use a more restricted scale with five levels: 1 is equal importance, 3 is moderate importance, 5 is strong importance, 7 is very strong or demonstrated importance and 9 is extreme importance. Because different application have different requirements, in this paper, we consider two types of application -- VoIP as a real-time application example and Media Content Downloads as a non-real-time application example. Tables 2 and 3 show the pair-wise relative importance of the decision criteria for the real-time and non real-time applications respectively.

\begin{tabular}{llll}
\hline Real-time & CINR & BW & Cong. delay \\
\hline CINR & $1 / 1$ & $5 / 1$ & $3 / 1$ \\
\hline BW & $1 / 5$ & $1 / 1$ & $1 / 7$ \\
\hline Cong. delay & $1 / 3$ & $7 / 1$ & $1 / 1$ \\
\hline
\end{tabular}

Table 2. Pair-wise Matrix for real-time application

\begin{tabular}{llll}
\hline Non Real-time & CINR & BW & Cong. delay \\
\hline CINR & $1 / 1$ & $3 / 1$ & $5 / 1$ \\
\hline BW & $1 / 3$ & $1 / 1$ & $7 / 1$ \\
\hline Cong. delay & $1 / 5$ & $1 / 7$ & $1 / 1$ \\
\hline
\end{tabular}

Table 3. Pair-wise Matrix for non real-time application

Based on the AHP procedure explained earlier the weighting vector for the real time application and non real-time applications are as shown in equations (17), and (18).

$$
\begin{aligned}
& w_{r}=\left[\begin{array}{lll}
0.6186 & 0.0662 & 0.3149
\end{array}\right] \\
& w_{n r}=\left[\begin{array}{lll}
0.6186 & 0.3149 & 0.0662
\end{array}\right]
\end{aligned}
$$

\subsection{Alternative ranking using TOPSIS}

After weighting the decision criteria using the AHP method, the TOPSIS method will be used to rank the alteratives available for the Target Base Station (TBS), and then choose the highest 
alterative score is chosen for the TBS. As mentioned earlier, TOPSIS concept is that the chosen alternative should have the shortest distance from the positive ideal solution (PIS) and the farthest from the negative. More explanation about this will be discussed in details in the numerical analysis section.

\section{Numerical analysis}

In this section a numerical analysis of the proposed scheme is performed, and then compared with the Weighted Sum Method (WSM) and conventional signal strength based cell selection. The criteria weighting based on user application requirements have been calculated previously, as shown in equation (17) and (18) for real-time and non real-time applications respectively.

Suppose that we have three candidate TBSs as shown in Figure 1. After the information collection stage, the MS will maintain the criteria values in a decision matrix, $\operatorname{Dm}(A)$. The decision matrix consists of three criteria, viz CINR in $\mathrm{dB}$, BW in Kbps and congestion delay in ms for three candidate BSs as shown in (19).

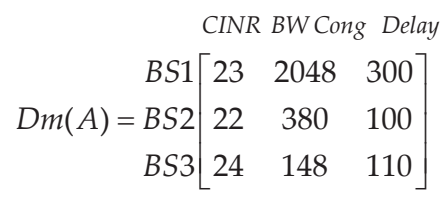

Using the TOPSIS method, $\operatorname{Dm}(A)$ first has to be normalized first using equation (7), because the criteria use different units. The normalized matrix is shown in (20).

$$
\operatorname{Dm}\left(A^{\prime}\right)=B S 2\left[\begin{array}{lll}
0.5770 & 0.9807 & 0.8960 \\
0.5519 & 0.1820 & 0.2987 \\
0.6021 & 0.0709 & 0.3285
\end{array}\right]
$$

After getting the normalized decision matrix, it is weighted using by multiplying it with the weighting factor. Two types of application have been considered, therefore, we have two weighting factors, which will produce two weighted decision matrices.

For the real-time application, the weighted decision matrix is as shown in equation (21).

$$
\operatorname{Dm}\left(A^{\prime}\right)_{r}=B S 2\left[\begin{array}{lll}
0.3569 & 0.0649 & 0.2822 \\
0.3414 & 0.0120 & 0.0941 \\
0.3724 & 0.0047 & 0.1035
\end{array}\right]
$$


For the non real-time application, the weighted decision matrix is as shown in equation (22).

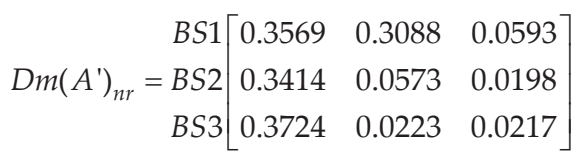

To calculate the distance from the positive ideal solution (PIS) and the farthest from the negative ideal solution (NIS), equations (8) - (11) are used. CINR and BW are considered as benefit criteria and congestion delay is considered as a cost criteria. The ranking of the alternative TBSs for real-time and non real-time application are shown in equation (23) and (24) for real time and non real time application respectively.

$$
\begin{array}{ccc}
B S 1 & B S 2 & B S 3 \\
R_{r}=\left[\begin{array}{lll}
0.2479 & 0.7543 & 0.7484
\end{array}\right] \\
R_{n r}=\left[\begin{array}{ccc}
0.8710 & 0.1724 & 0.1454
\end{array}\right]
\end{array}
$$

\begin{tabular}{|c|c|c|c|c|c|}
\hline \multirow[t]{2}{*}{ MCDM methods } & \multicolumn{2}{|c|}{ WSM } & \multicolumn{3}{|c|}{ TOPSIS } \\
\hline & BS1 & BS3 & BS1 & BS2 & BS3 \\
\hline VolP(real-time) & \multicolumn{2}{|c|}{$[0.7640,0.8942,0.9097]$} & \multicolumn{3}{|c|}{$[0.2479,0.7543,0.7484]$} \\
\hline Media Content Download (non real-time) & \multicolumn{2}{|c|}{$[0.9298,0.6917,0.7015]$} & \multicolumn{3}{|c|}{$[0.8710,0.1724,0.1454]$} \\
\hline
\end{tabular}

Table 4 shows the summary of the ranking of the available alternative TBSs using TOPSIS compared with WSM and also illustrated in Figures 4 and 5 respectively.

Table 4. Ranking of the available alternatives using WSM and TOPSIS

Using other wayS of viewing the cell selection results, Table 5 compare between TOPSIS, WSM and conventional signal strength-based cell selection.

\begin{tabular}{lccc}
\hline & Signal strength & WSM & TOPSIS \\
\hline VolP & BS3 & BS3 & BS2 \\
\hline Media Content Download & BS3 & BS1 & BS1 \\
\hline
\end{tabular}

Table 5. Selected cell using TOPSIS, WSM and the conventional signal strength schemes 
For real-time applications, WSM ranks B3 as the best, and TOPSIS ranks B2 as the best. Both two results are reasonable, because all of them have good CINR and small delay. However, TOPSIS ranks B2 as the best, because it provides the lowest delay. So the TOPSIS looks like more sensitive to the criteria weighting. The conventional signal strength scheme ranks B3 as the best, because it provides the best CINR.

For the non real-time application, both WSM and TOPSIS ranks B1 as the best, because it has the highest BW while the conventional signal strength scheme ranks B3 as the best; although it has the best CINR, It provides the worst BW, which is an important criteria in the Media Content Download application.

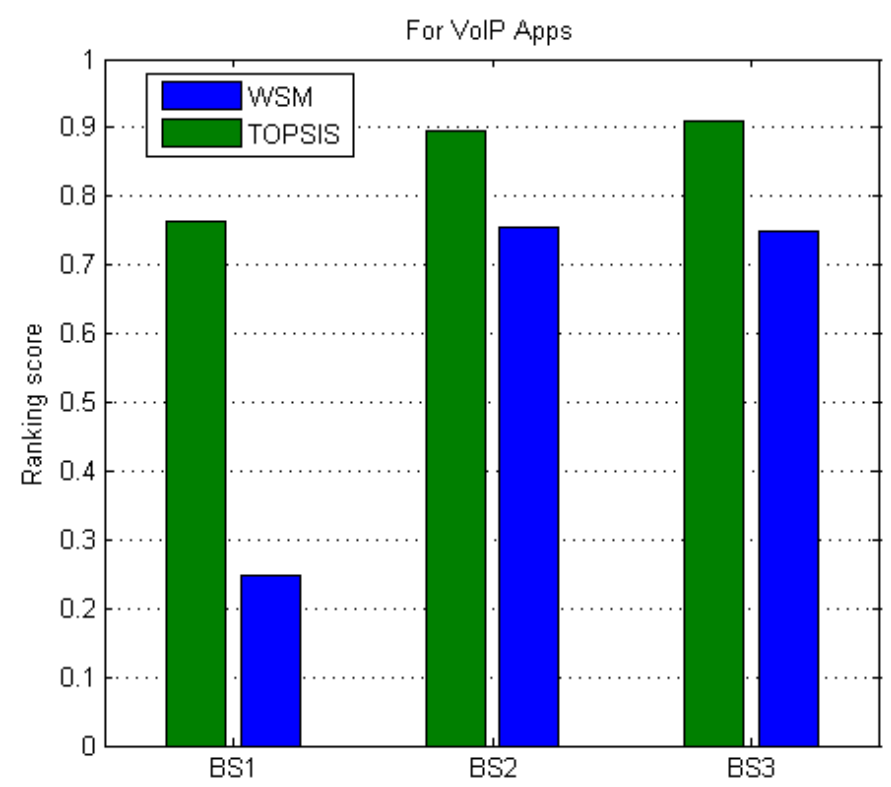

Figure 4. The WSM and TOPSIS scores for real-time applications

From table 4, we can see that application requirement or the weighting factor has an influence on the ranking order. As mentioned before, both WSM and TOPSIS have different ranking orders for VoIP as a real-time applicatipn. This is further exemplified by the sensitivity analysis on the weighting factor in Figure 6 and Figure 7 respectively.

When the congention delay criteria weighting is changed, and other parameters are kept constant we found that the ranking result is more sensitive when TOPSIS method is used. For example, in Figure 6 using WSM method the BS2 score changes form 0.6 to 0.95 when the congestion delay creiteria weighting is changed, so the range of the score change is 0.35 . Whereas in Figure 7 using TOPSIS method when the congention delay criteria weighing is changed, the BS2 score changes from 0.18 to 0.92 , so the range of the score change is 0.74 , and form this it is clear that TOPSIS method is more sensitive to the weighting factor. 


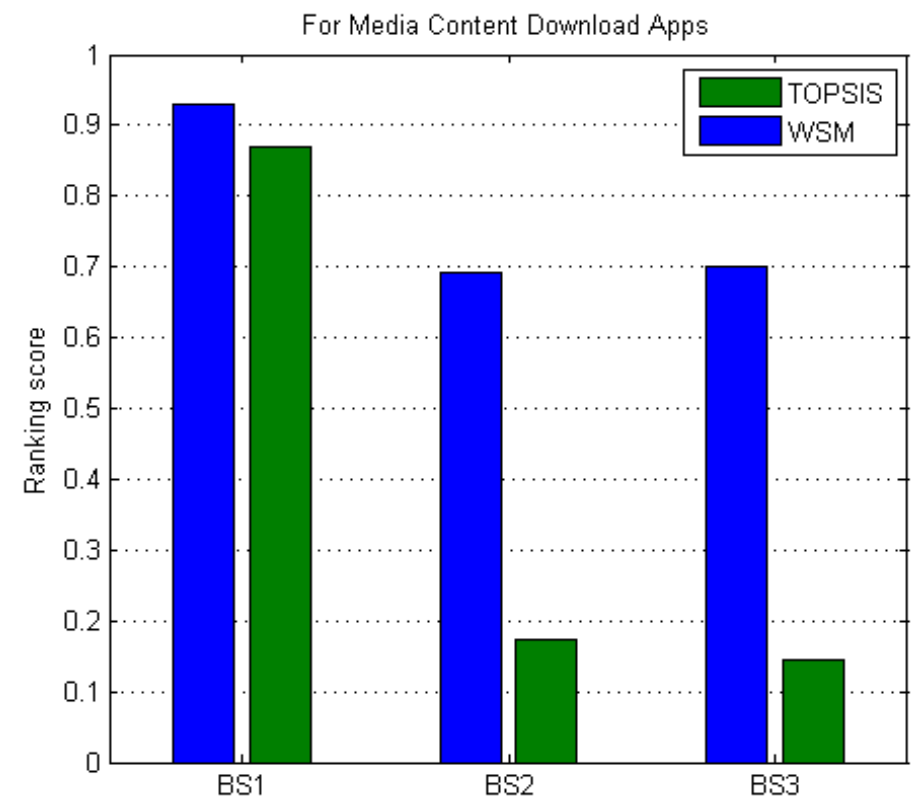

Figure 5. The WSM and TOPSIS scores for the non- real-time application

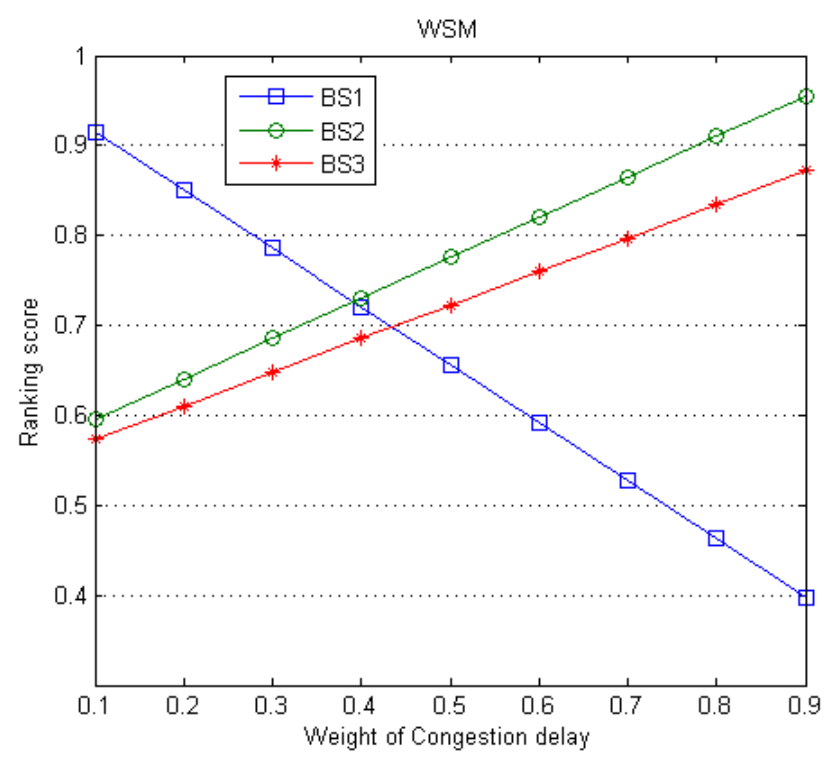

Figure 6. Sensitivity of congestion delay weighting using WSM 


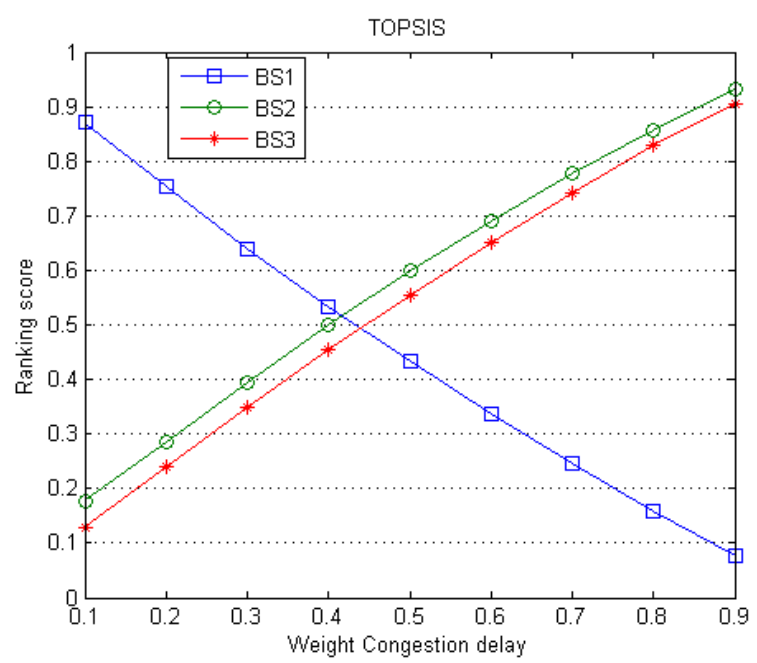

Figure 7. Sensitivity of congestion delay weighting using TOPSIS

\section{Conclusion}

Mobile WiMAX is one of the 3G/4G broadband wireless technology network that is capable of delivering triple play (voice, data, and video) services. The variety of user application needs different network requirements. So, handover mechanism and the cell selection scheme have to be efficient to meet the application need during and after the handover process. This paper described a smart way of TBS selection. It is based on multi-criteria based selection to meet the user requirements during handover. We proposed the AHP method for criteria weighting and TOPSIS for alternative BSs ranking as a multi-criteria decision-making selection scheme to meet the MS application requirements. The proposed, Hybrid AHP and TOPSIS-based Cell Selection (HATSC) scheme is based on multi-criteria such as CINR, bandwidth (BW) and congestion delay to select the TBS. Numerical analysis shows that TOPSIS is more sensitive to criteria weighting factor, and WSM gives a relative conservative ranking result, and both of them results in better performance than the conventional signal strength scheme.

\section{Author details}

Mohammed A. Ben-Mubarak, Borhanuddin Mohd. Ali, Nor Kamariah Noordin, Alyani Ismail and Chee Kyun $\mathrm{Ng}$

Department of Computer and Communication Systems Engineering, Faculty of Engineering, Universiti Putra Malaysia, Selangor, Malaysia 


\section{References}

[1] Mohamed, M, Zaki, F, \& Mosbah, R. Improving Quality of VoIP over WiMAX", IJCSI International Journal of Computer Science Issues, (2012). , 9(3), 85-91.

[2] WiMAX Forum members. Mobile WiMAX- Part I: A Technical Overview and Performance Evaluation", March [(2006).

[3] Ben-mubarak, M, Ali, B. M, Noordin, N. K, Ismail, A, \& Ng, C. K. Review of Handover Mechanisms to Support Triple Play in Mobile WiMAX". IETE Tech Rev, (2009). , 258-267.

[4] Ben-mubarak, M, Ali, B. M, Noordin, N. K, Ismail, A, \& Ng, C. K. Movement Direction based-Handover Scanning for Mobile WiMAX", the 17Asia-Pacific Conference on Communication (APCC 2011), Sabah-Malaysia, Oct. (2011). , 2-5.

[5] IEEE Standard for Local and metropolitan area networks Part 16: Air Interface for Fixed and Mobile Broadband Wireless Access Systems. (2006).

[6] Choi, S, Hwang, G, Kwon, T, Lim, A, \& Cho, D. Fast Handover Scheme for Real-Time Downlink Services in IEEE 802.16e BWA System". Proceeding of IEEE Vehicular Technology Conference, (2005). , 2028-2032.

[7] Chen, L, Cai, X, Sofia, R, Huang, Z, \& Cross-layer, A. Fast Handover Scheme for Mobile WiMAX". Proceeding of IEEE Vehicular Technology Conference, Baltimore, MD, (2007). , 1578-1582.

[8] Chen, J, Wang, C, \& Lee, J. Pre-Coordination Mechanism for Fast Handover in WiMAX Networks". Proceeding of IEEE (AusWireless2007), Sydney, Australia, (2007). , 15-20.

[9] Jiao, W, Jiang, P, \& Ma, Y. Fast Handover Scheme for Real-Time Applications in Mobile WiMAX". Proceeding of IEEE International Conference on Communications (ICC'07), Glasgow, Scotland, (2007). , 6038-6042.

[10] Yeh, J, Chen, J, \& Agrawal, P. Fast Intra-Network and Cross-Layer Handover (FINCH) for WiMAX and Mobile Internet. IEEE Transactions on Mobile Computing, (2009). , 558-574.

[11] Gu, S, \& Wang, J. An enhanced handover target cell selection algorithm for WiMAX network," 15th Asia-Pacific Conference on Communications (APCC), (2009). , 774-777.

[12] Yang, S, Wu, J, \& Huang, H. A vertical Media-Independent Handover decision algorithm across Wi-Fi and WiMAX networks", 5th IFIP International Conference on Wireless and Optical Communications Networks, (2008). , 1-5. 
[13] Wu, J, Yang, S, Hwang, B, \& Terminal-controlled, A. vertical handover decision scheme in IEEE 802.21-enabled heterogeneous wireless networks", Int. J. Commun. Syst. (2009). , 819-834.

[14] Triantaphyllou, E, Shu, B, Sanchez, N, \& Ray, T. Multi-criteria decision making: an operations research approach", Encyclopedia of Electrical and Electronics Engineering. New York: John Wiley \& Sons, (1998). , 15, 175-186.

[15] Yap, K. All-IP 4G Mobile Networks and Beyond", WINLAB Seminar, Rutgers University, North Brunswick, (2010).

[16] Yap, K, Katti, S, Parulkar, G, \& Mckeown, N. Deciphering a Commercial WiMAX Deployment using COTS Equipments", Stanford University, Technical Report, (2010).

[17] Straccia, U. Multi-criteria decision making in fuzzy Description Logics: A First step", In Proceedings of the 13th International Conference on Knowledge-Based \& Intelligent Information \& Engineering Systems, Lecture Notes in Artificial Intelligence, Springer, (2009). , 79-87.

[18] Ramanathan, R. A note on the use of the analytical hierarchy process for environmental impact assessment", J Environ Manag (2001). , 27-35. 
